\title{
Value of ambulatory ST segment monitoring in patients with chronic stable angina: Does measurement of the "total ischaemic burden" assist with management?
}

Royal Brompton National Heart and Lung Hospital, London D Mulcahy

J Parameshwar

D Holdright

C Wright

J Sparrow

$\mathrm{K} M$ Fox

Hillingdon Hospital, Uxbridge, Middlesex G Sutton

Correspondence to Dr David Mulcahy, Roya Brompton National Heart and Lung Hospital, Sydney Street, London SW3 6NP Accepted for publication 3 November 1991

\begin{abstract}
Objective-To assess the prognostic significance of transient ischaemic episodes during daily activities in patients with stable angina.
\end{abstract}

Patients and methods-172 patients with stable angina attending the cardiac outpatients departments of Hillingdon Hospital ( $n=155)$ and the National Heart Hospital $(n=17)$ were prospectively studied by exercise testing and 48 hours of ambulatory ST segment monitoring, and followed for prognostic purposes for up to 39 months (mean 24.5 months). Patient inclusion depended on a clinical diagnosis of stable coronary artery disease which necessitated outpatient review (and antianginal treatment in $94 \%$ of patients). It was not dependent on objective evidence of reversible ischaemia. Events recorded during the follow up period included death, non-fatal myocardial infarction, unstable angina, and the requirement for revascularisation.

Results-72 patients (42\%) had transient ischaemic episodes during daily activities, and 104 patients $(60.5 \%)$ had an ischaemic response to exercise. 63 patients $(36 \%)$ had evidence of ischaemia on both investigations; with $59(34 \%)$ having no documented ischaemia on either investigation. There were 27 patient events $(15 \cdot 7 \%)$ recorded over a mean 24.5 month follow up, including five deaths $(2.9 \%)$ (three cardiac related (1.7\%)), six non-fatal myocardial infarctions (3.5\%), six admissions with unstable angina $(3.5 \%)$, and 10 revascularisation procedures $(5 \cdot 8 \%)$. Of the nine "hard" or objective end points (cardiac death and non-fatal myocardial infarction), only two had evidence of transient ischaemia on ambulatory ST segment monitoring at initial investigation, with 10 of the 25 patients $(38.5 \%)$ with any cardiac event having such episodes.

Conclusions-The outcome in patients with chronic stable angina receiving standard medical treatment was good over a mean two year follow up period. For the purpose of assessing prognosis over this time scale, there was no advantage to performing ambulatory ST segment monitoring in such patients.
Many studies have confirmed that a significant proportion of patients with stable angina have transient ischaemic episodes during their daily activities and that most of these episodes occur in the absence of symptoms. ${ }^{1-3}$ It has been shown that the characteristics of silent and painful episodes of transient ischaemia are similar. ${ }^{45}$ Though there is considerable evidence to suggest that transient ischaemia (predominantly silent) is of adverse prognostic significance in patients with unstable angina ${ }^{67}$ and in high risk patients after myocardial infarction, ${ }^{8}$ there is little information on the prognostic significance of transient ischaemic episodes in patients with chronic stable angina. Two groups have recently reported that the demonstration of transient ischaemia during the daily activities of patients with stable angina helps to identify subgroups at increased risk of developing the end points of coronary disease over a one to two year period; 910 however, these studies only included patients with documented evidence of ischaemia on exercise testing, usually at low workloads, and in one study ${ }^{10}$ a 23 month cardiac mortality of $15 \%$ was reported in patients with angina that was well controlled by treatment. Large scale studies, however, show that overall prognosis in chronic stable angina is generally better than this. ${ }^{1112}$

We assessed the prognostic significance of transient myocardial ischaemia in patients attending two cardiac outpatient departments with a diagnosis of stable coronary artery disease, for which most had been prescribed suitable antianginal treatment. This population was chosen because it is typical of most patients with angina being treated by their physician.

\section{Patients and methods}

One hundred and seventy two patients with stable angina who were attending the cardiac outpatient department of a district general hospital $(n=155)$ or a tertiary referral cardiac centre $(n=17)$ were included in the study between February 1988 and August 1989. All were enrolled during a routine outpatient visit. There were 148 men and 24 women (mean age 58.4 years (range $37-78$ ). Seventy five patients $(44 \%)$ had had a previous myocardial infarction and $23(13.4 \%)$ had undergone coronary artery bypass surgery $(n=21)$ or percutaneous transluminal coronary angioplasty 
$(n=2)$. A total of 141 patients were taking long-acting antianginal medications.

Patients were included in the study if they had stable coronary artery disease which necessitated regular outpatient review. Documented evidence of ischaemia was not a requirement for inclusion, nor was absolute evidence of coronary artery disease. Exclusion criteria included the presence of significant conduction disturbances and medical treatment likely to affect the ST segment.

\section{INVESTIGATIONS}

All patients were referred to the National Heart Hospital, where they underwent maximal symptom limited treadmill exercise testing followed by 48 hours of ambulatory ST segment monitoring, which was performed outside the hospital.

\section{Exercise testing}

All patients underwent maximal symptom limited treadmill exercise testing according to the modified Bruce protocol. ${ }^{13}$ Electrocardiograms were recorded at the onset and every three minutes during exercise and also when significant ST segment depression developed. A test was regarded as positive for ischaemia if there was planar or downsloping ST segment depression of $\geqslant 1 \mathrm{~mm}$ from baseline measured $0.08 \mathrm{~s}$ after the J point, that persisted for three consecutive beats in one or more leads. Exercise was limited by the development of chest pain, breathlessness, exhaustion, intermittent claudication, ventricular arrhythmias, or hypotension.

\section{Ambulatory ST segment monitoring}

All patients underwent 48 hours of ambulatory ST segment monitoring outside hospital using pregelled electrodes to record two bipolar leads, an anterior lead (CM5), and an inferior lead. The sites and method of application of these electrodes have been described elsewhere. ${ }^{14}$ Two channel recordings were then made onto magnetic tape with a frequency modulated dual channel recorder (Oxford Medilog 2 or MR35). These systems have an adequate frequency response to record and display accurately the ST segment. Tapes were then visually analysed at 60 to 120 times normal speed by an Oxford Medilog MA20 scanner, and all printouts were at $25 \mathrm{~mm} / \mathrm{s}$. Significant ST segment depression was defined as a planar or downsloping shift of the ST segment of $\geqslant 1 \mathrm{~mm}$ measured $0.08 \mathrm{~s}$ after the $\mathrm{J}$ point and persisting for more than one minute. Significant ST segment elevation was defined as an upward shift of $>1 \mathrm{~mm}$ of the ST segment measured at the $J$ point and persisting for more than one minute. Changes in the $T$ wave vector were not regarded as evidence of myocardial ischaemia unless they were accompanied by significant ST segment changes.

During the period of ambulatory monitoring all patients maintained a detailed angina diary, recording the time of each episode of pain, the activity at the onset of symptoms, and the requirement for glyceryl trinitrate. An event marker on the ambulatory monitor was pressed at the onset of symptoms. This marked the magnetic tape so that the electrocardiographic playback would automatically display the electrocardiogram at that time. All patients were encouraged to continue with their normal daily activities during the monitoring period.

\section{ANTI-ANGINAL MEDICATIONS}

Patients received standard antianginal medications as prescribed by their attending cardiologist during the period of study. One hundred and forty one patients $(82 \%)$ received long-acting antianginal treatment. This consisted of a $\beta$ adrenergic blocking agent $(n=109)$, a calcium channel blocking agent $(n=60)$, and long-acting nitrates $(n=88)$, either singly $(n=54)$ or in various combinations (double therapy $n=59$; triple therapy $n=28$; total $\mathrm{n}=87)$. A further 20 patients $(11 \cdot 6 \%)$ used short-acting nitrate preparations as required, and 11 patients $(6.4 \%)$ reported not taking any medications at the time of study.

\section{FOLLOW-UP DATA}

Of the 172 patients enrolled in the study, all were reviewed as outpatients as near to or more than two years after inclusion where possible. Where outpatient follow up was not possible, details of patient deaths or clinical state were obtained from the general practitioner or by telephone interview with the patients. For those with events, follow up was taken as the time of occurrence of such episodes. Details of hospital admissions and clinical state over the follow up period were recorded in each case, and a resting electrocardiogram was performed for reasons of comparison to exclude "silent myocardial infarction" over the intervening time. All reported non-fatal myocardial infarctions were confirmed by assessment of the clinical notes and the electrocardiograms and by confirmation of a significant rise (more than twice normal) in cardiac enzymes. Silent myocardial infarction was sought in 138 patients without a recorded event during the follow up period who were reviewed as outpatients.

\section{STATISTICAL ANALYSIS}

The three variables (exercise duration, time to angina, and time to ischaemia on exercise) were compared by a one way analysis of variance. Statistical significance was taken to be $\mathrm{p}<0.05$.

\section{Results}

One hundred and seventy two patients were studied on an intention to treat basis: 155 $(90 \%)$ had evidence of coronary artery disease, with reversible ischaemia on exercise testing or ambulatory ST segment monitoring, or prior myocardial infarction, cardiac catheterisation, coronary artery bypass surgery, or coronary angioplasty. A further 17 patients had chest pain suggestive of angina, without having objective evidence of reversible ischaemia.

\section{EXERCISE TESTING}

One hundred and seventy two patients underwent maximal symptom limited treadmill exer- 
cise testing: $104(60.5 \%)$ had an ischaemic response. The mean total duration of exercise for the whole group was 8.4 minutes, the mean time to ischaemia was 6.3 minutes, and the mean time to angina was 6.4 minutes. One hundred and twelve (65\%) patients developed angina during the investigation.

\section{AMBULATORY ST SEGMENT MONITORING}

During a total of 7568 hours of ambulatory ST segment monitoring in 172 patients (mean 44 hours), 72 patients $(42 \%)$ had one or more episodes of transient ST segment change. There were 370 episodes of ischaemia recorded, of which $271(73 \%)$ were silent and 99 (27\%) painful. Of the 72 patients, $32(44 \%)$ had only silent episodes, $14(19 \%)$ only painful episodes, and $26(36 \%)$ had both silent and painful episodes.

Figure 1 shows the relation between the results of exercise testing and ambulatory ST segment monitoring. Sixty three patients with ischaemia on ambulatory ST segment monitoring had an ischaemic response during exercise and only nine patients had a negative exercise test but transient episodes of ischaemia during daily activities. Forty one patients had a positive exercise test and no ambulatory ischaemia. A total of 59 patients had no evidence of ischaemia on either investigation. Patients with both tests positive $(n=63)$ had a significantly shorter time to ischaemia on exercise testing than those with only a positive exercise test $(\mathrm{n}=41)(5.91$ v $7.23 \mathrm{~min}$, $\mathrm{p}<0.02)$. Those with both tests positive had a similar maximal exercise time as those with ischaemia only on exercise $(7.73 v 8.64 \mathrm{~min}$, $p=0.088$ ). The difference in time to maximal exercise between groups with both tests positive $(n=63)$ and both tests negative $(n=59)$ for ischaemia was statistically significant $(7.73 v 8.92 \mathrm{~min}, \mathrm{p}<0.03)$.

\section{RELATION OF ISCHAEMIA TO SYMPTOMS ON} EXERCISE

One hundred and twelve patients (65\%) experienced angina during formal exercise testing. Fifty $(80 \%)$ of 63 patients with ischaemia both during exercise and daily activities, and 34 $(83 \%)$ of 41 patients with ischaemia only on exercise testing developed angina during exercise compared with $24(40 \%)$ of 59 patients

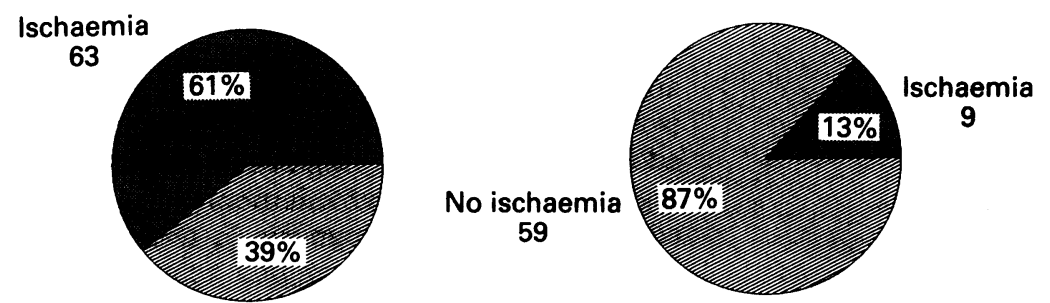

No ischaemia
41

Positive exercise test $(N=104)$
Negative exercise test $(N=68)$
Figure 1 Number of patients with evidence of transient ischaemic change during daily Figure 1 Number of patients with evidence of transient ischaemic change during daily
life in association with $(n=104)$ and without $(n=68)$ a positive exercise test for ischaemia. with no evidence of ischaemia on either investigation.

\section{FOLLOW UP DATA}

Patients were followed up for a mean of 24.5 months (range 1-39 months), during which time 27 patient events were recorded, of which $25(14 \cdot 7 \%)$ were cardiac. These are divided into "hard" or objective end points (non-fatal myocardial infarction and cardiac death) and "soft" or subjective end points (unstable angina and revascularisation) where physician diagnosis and decision making influence the event rate.

There were five deaths recorded, three from heart disease and two from malignancy. Six patients had an acute myocardial infarction. A further six patients were admitted to hospital where unstable angina was diagnosed, and 10 patients were referred for revascularisation procedures (bypass $n=9$, angioplasty $n=1$ ), of which nine were performed electively (fig 2).

\section{EVENTS RECORDED}

\section{Hard end points}

Nine objective end points were recorded (three cardiac deaths and six acute myocardial infarctions) during the 24 month follow up period; four patients showed evidence of ischaemic change on exercise testing, but only two had such evidence during ambulatory monitoring. One patient who died suddenly while driving his car had had frequent episodes of ischaemia during ambulatory monitoring and an ischaemic exercise test, and a further patient with ischaemic changes during both investigations had a non-fatal myocardial infarction. Five of the six patients with non-fatal myocardial infarction had no evidence of ischaemia on either investigation at inclusion in the study.

\section{Soft end points}

Six patients were admitted to hospital with unstable angina: four had had a positive exercise test and two had positive tapes, with five of the six having evidence of ischaemia on at least one investigation. Of the 10 patients referred for revascularisation because of symptoms, seven had a positive exercise test and six had positive tapes. Seven had evidence of ischaemia on at least one investigation.

\section{RELATION OF COMBINED ELECTRO}

CARDIOGRAPHIC FINDINGS TO EVENTS

There were 63 patients with a positive exercise test and evidence of transient ischaemia on ambulatory ST segment monitoring; one patient died and one had acute non-fatal myocardial infarction over the two years of follow up (objective event rate $3 \cdot 2 \%$ ). A further patient had unstable angina, and six were referred for revascularisation (total nine patient events $(14.5 \%))$. Of the 59 patients with neither test positive, five had an acute non-fatal myocardial infarction (objective event rate $8.5 \%$ ), one unstable angina, and three were referred for revascularisation (total nine patient events $(15 \cdot 2 \%)$ ). A further two deaths, four episodes of unstable angina, and one referral for revascularisation occurred in those 50 


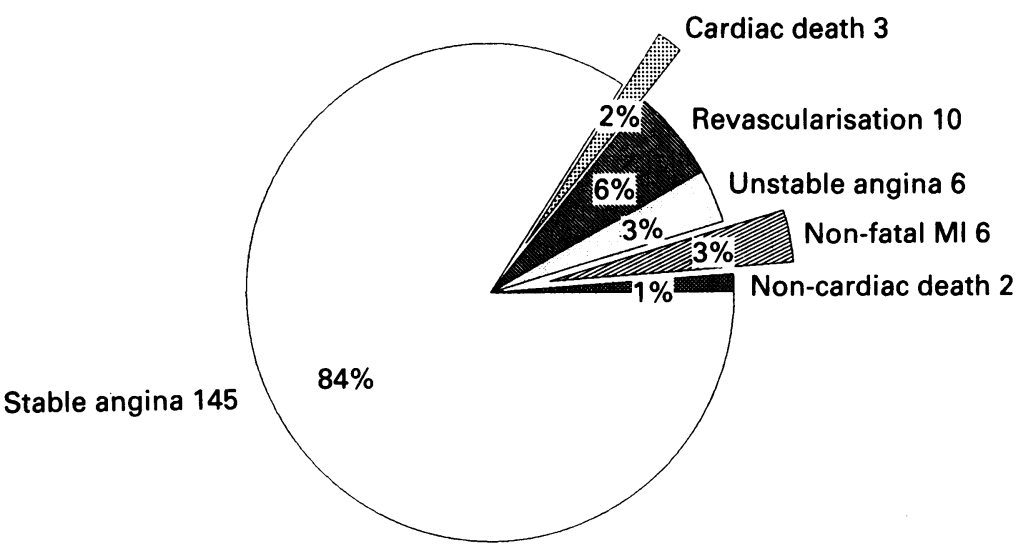

Figure 2 Recorded events in 172 patients with stable coronary artery disease over a 24.5 month follow-up.

\section{Discussion}

This study confirms that approximately $40 \%$ of patients with chronic stable angina receiving standard medical treatment, who were not selected for study on the basis of exercise test results, had episodes of transient myocardial ischaemia during their daily lives, most of $I$ which were silent, ${ }^{15}$ and it also confirms that exercise testing is a more sensitive investigation $\vec{C}$ than ambulatory ST segment monitoring for the detection of coronary artery disease. 91016 We chose to assess the total ischaemic burden rather than to highlight the significance of silent ischaemia, because silent and painful ischaemia have similar characteristics. ${ }^{45}$ It is clear that the frequency of such transient ischaemic changes is relatively low in an ambulant population such as this, with only 370 episodes of ischaemia in total being recorded during more than 7500 hours of monitoring during daily activities.

This study also confirms that the medium term prognosis for "unselected" patients with stable angina in the population is very good, with a two year all cause mortality of approximately $3 \%$ (cardiac mortality of $1.7 \%$ ) and an incidence of acute non-fatal myocardial infarction of $3.5 \%$. These figures accord with large prognostic studies in patients with stable angina. ${ }^{11} 12$ Brunelli et $a l^{11}$ recently reported on a mean 66 month follow up of 1083 medically treated patients with ischaemic heart disease. Seventy seven per cent had a positive exercise test for ischaemia and $74 \%$ had documented significant coronary artery disease: $77 \%$ were receiving routine antianginal treatment. They reported an overall annual cardiac mortality rate of $1.54 \%$, rising to $4.1 \%$ in those with three vessel disease and up to $5.7 \%$ in those with three vessel disease and poor left ventricular function. In the coronary artery surgery study (CASS study), ${ }^{12}$ which included assessment of survival in medically treated patients with class I and II stable angina, total annual mortality was $1.6 \%$, rising to $4.0 \%$ in the subgroup with three vessel disease and poor left ventricular function.

PROGNOSTIC SIGNIFICANCE OF THE TOTAL ISCHAEMIC BURDEN IN STABLE ANGINA Previous reports

Recent studies have reported on the prognostic significance of transient episodes of silent $\sigma$ ischaemia in stable angina. ${ }^{1017}$ Rocco and coworkers in a study of 86 patients with $\stackrel{\odot}{\stackrel{C}{C}}$ documented coronary artery disease, stable $\stackrel{\mathscr{P}}{?}$ angina, and a positive exercise test for 0 ischaemia, reported that those patients with

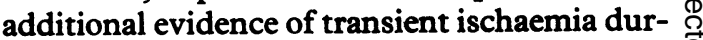
ing ambulatory ST segment monitoring had an $\stackrel{\varnothing}{\varnothing}$ adverse short-term prognosis in terms of the development of unstable angina, acute myocar- $\delta$ dial infarction, death, or the requirement for revascularisation compared with those without evidence of transient ischaemia. ${ }^{9}$ Of the 21 end points recorded (15 unstable angina or revascularisation) during the 12.5 month follow up period, 20 occurred in the $49^{-}$patients with transient ischaemic episodes.

Deedwania and Carbajal reported a two year Figure 3 Number of patients with stable angina and frequency of hard (non-fatal revascularisation) end points over a 24.5 month follow up in those patients with evidence of ischaemia on both exercise testing and ambulatory ST segment monitoring, on neither test, and on one test only.

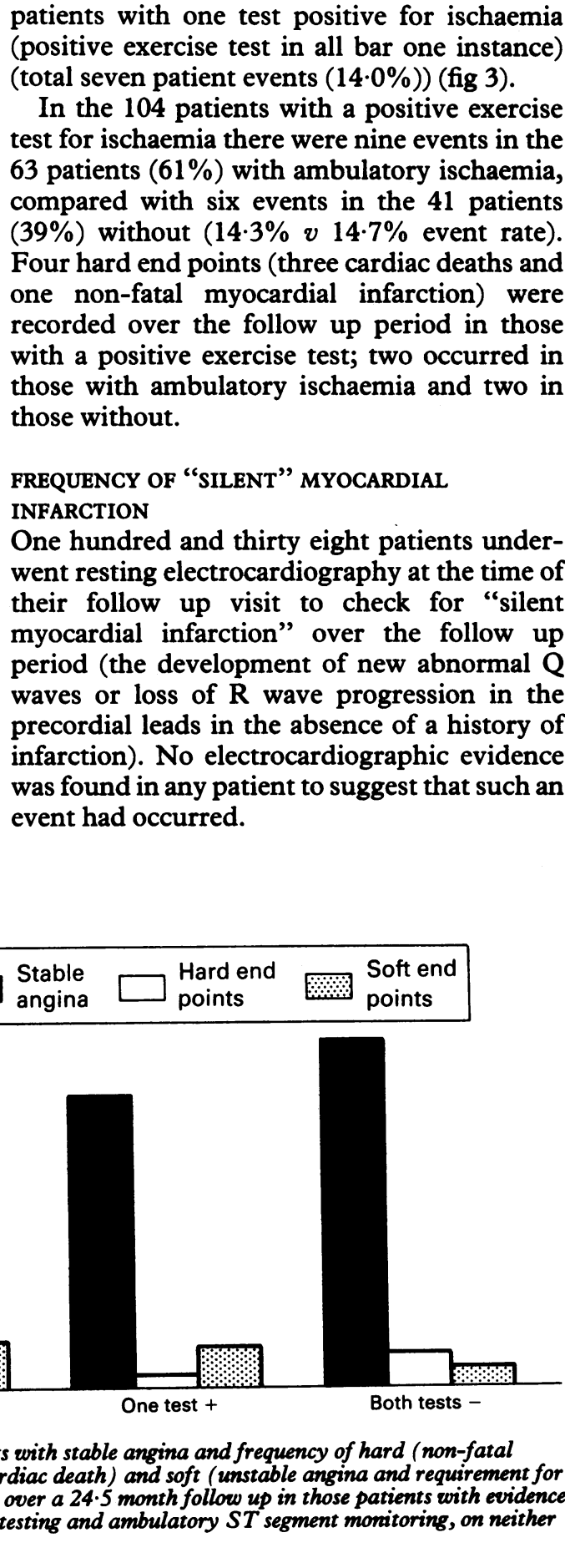


follow up study to assess the prognostic significance of silent ischaemia during daily life for mortality in patients with stable angina. ${ }^{10}$ There were 107 patients with long term stable angina in whom symptoms were controlled by standard treatment; $46(43 \%)$ of these had evidence of transient ischaemia during ambulatory monitoring. There were 16 cardiac deaths recorded during the 23 month follow up (three further non-cardiac deaths); 11 occurred in the 46 patients with transient ischaemia during daily life. This represents a $24 \%$ cardiac mortality over the follow up period in those with transient ischaemia on ambulatory monitoring and a $15 \%$ cardiac mortality for the total group. In the above quoted studies, mean time to ischaemia on exercise testing was $2.9 \mathrm{~min}$ utes $^{9}$ and 3.3 minutes $^{10}$ in those patients who also had changes during ambulatory monitoring, thus they represent highly selected groups of patients.

A further study by Aronow and Epstein reported that $62(34 \%)$ of 185 elderly patients (mean age 82 years) with coronary artery disease had silent ischaemia during daily life and that $65 \%$ of such subjects ( 40 of 62 patients) developed non-fatal or fatal myocardial infarction, ventricular fibrillation, or sudden cardiac death during just over two years of follow up. ${ }^{17}$

The event rates in these studies ${ }^{910}$ seem high in a group of stable angina patients apparently well controlled by medical treatment, and the results may not be representative of patients with angina in general. Deedwania and Carbajal point out that the vast majority of their patients were unable to complete stage two of the Bruce protocol and might represent a group at higher risk of silent ischaemia and subsequent mortality. ${ }^{10}$ Our event rate corresponds with other reports in populations with stable angina, however, it is perhaps surprising that $78 \%$ of hard end points occurred in patients without documented evidence of ischaemia during daily life. The apparent lack of association between transient myocardial ischaemia and non-fatal myocardial infarction may be explained in part by the fact that acute myocardial infarction can often result from acute plaque rupture of what were small or medium sized lesions, insufficient to cause prior ischaemic changes. These findings do emphasise the importance of including unselected patients in prognostic angina studies, otherwise the results from highly selected populations may be inappropriately extrapolated to patients with angina in general. While others have shown that silent ischaemia and the total ischaemic burden carries prognostic weight within a subselected group of angina patients who have a demonstrated propensity to exercise ischaemia, usually at low workloads ${ }^{910}$ we were unable to bear this out in the present study, with only two of 63 patients with both exercise and ambulatory ischaemia dying or having a non-fatal myocardial infarction over a two year period compared with five of 59 patients without evidence of ischaemia having non-fatal myocardial infarction.

Two recent studies have assessed the prognostic significance of ambulatory ischaemia in the stable phase after myocardial infarction ${ }^{18}$ and after coronary artery bypass surgery. ${ }^{19}$
Tzivoni et al studied 224 low risk post-infarction patients, performing ambulatory ST segment monitoring and exercise testing at a mean of two years after infarction. They then followed up these patients for a mean of 28 months. ${ }^{18}$ Thirty three per cent (74 patients) had transient ischaemia during ambulatory monitoring (all also had a positive exercise test) and $51 \%$ of these had a "cardiac event" during the follow up period compared with $12 \%$ of those without transient ischaemia ( $p<$ $0.0001)$. There was no difference, however, between groups with and without transient ischaemia in terms of the objective end points of cardiac death and acute non-fatal myocardial infarction, the differences being generated by an increased tendency for patients with transient ischaemia to become more symptomatic. These results imply that those with ischaemia on exercise testing and during daily life are more likely to develop symptoms requiring hospital admission or non-medical intervention (subjective end points chosen by physician), but are not more likely to experience a hard end point of coronary artery disease. In the present study $60 \%$ of patients referred for intervention because of symptoms had ischaemia on both investigations, thus supporting the findings of Tzivoni et al. ${ }^{18}$

Kennedy et al also performed ambulatory ST segment monitoring on 184 patients 12 months after coronary artery bypass surgery and noted that at a mean of four years after investigation the presence of transient silent ischaemic episodes had not helped to identify a group more likely to have cardiac events. ${ }^{19}$

\section{POTENTIAL LIMITATIONS OF STUDY}

We did not undertake coronary angiography in all patients included in this study and therefore have to accept that all patients may not have had coronary artery disease. To perform a clinically relevant study, however, we included patients on an "intention to treat" basis. In support of this, five of the six non-fatal myocardial infarctions occurred in those without objective evidence of reversible ischaemia.

In an unselected group of patients with stable angina, the two year outcome was favourable, and over this time the results of ambulatory ST segment monitoring seemed to contribute little to the identification of patients liable to have non-fatal myocardial infarction or cardiac death. While assessment of the total ischaemic burden may contribute prognostic information in subsets of patients with coronary artery disease, its true prognostic value in ambulant populations with stable angina must be assessed by performing large medium and long term studies of angina patients on an intention to treat basis, whether or not there is objective evidence of ischaemia. Only then would we be able to establish the precise importance of assessment of silent ischaemia and the total ischaemic burden in the general population of angina patients.

1 Stern S, Tzivoni D. Early detection of silent ischaemic heart disease by 24-hour electrocardiographic monitoring of disease by 24-hour electrocardiographic

2 Deanfield JE, Selwyn AP, Chierchia S, et al. Myocardial ischaemia during daily life in patients with stable angina: ts relation to symptoms and heart rate changes. Lancet 1983;i:753-8. 
3 Cecchi AC, Dovellini EV, Marchi F, Pucci P, Santoro GM, Fazzini PF. Silent myocardial ischemia during ambulatory electrocardiographic monitoring in patients with effort angina. J Am Coll Cardiol 1983;1:934-9.

4 Stern S, Gavish A, Weisz G, Benhorin J, Keren A, Tzivoni D. Characteristics of silent and symptomatic myocardial ischemia during daily activities. Am J Cardiol 1988; ischemia $61: 1223-8$.

5 Mulcahy D, Keegan J, Fox KM. Comparative characteristics of silent and painful ischemia during ambulatory monitoring in patients with coronary artery disease. Int Cardiol 1990;28:377-9.

6 Gottlieb SO, Weisfeldt ML, Ouyang P, Mellits ED, Gerstenblith G. Silent ischemia predicts infarction and death during two-year follow-up of unstable angina. J Am Coll Cardiol 1987;10:756-60.

7 Nademanee K, Intarachot V, Josephson MA, Rieders D, Mody FV, Singh BN. Prognostic significance of silent myocardial ischemia in patients with unstable angina. Am Coll Cardiol 1987;10:1-9.

8 Gottlieb SO, Gottlieb SH, Achuff SC, et al. Silent ischemia on Holter monitoring predicts mortality in high-risk poston Holter monitoring predicts mortality in high-risk pos

9 Rocco MB, Nabel EG, Campbell S, et al. Prognostic importance of myocardial ischemia detected by ambuimportance of myocardial ischemia detected by ambu-
latory monitoring in patients with stable coronary artery latory monitoring in patients with sta
disease. Circulation 1988;78:877-84.

10 Deedwania PC, Carbajal EV. Silent ischemia during daily life is an independent predictor of mortality in stable angina. Circulation 1990;81:748-56.

11 Brunelli C, Cristofani R, L'Abbate A. Long-term survival in medically treated patients with ischaemic heart disease and prognostic importance of clinical and electrocardiographic data. Eur Heart J 1989;10:292-303.

12 Vliestra RE, Frye RL, Kronmal RA, Sim DA, Tristani FE,
Killip III T, and the participants in the Coronary Artery Surgery Study. Risk factors and angiographic coronary artery disease: a report from the coronary artery surgery study (CASS). Circulation 1980;62:254-61.

13 Lipkin DP, Perrins J, Poole-Wilson PA. Respiratory gas exchange in the assessment of patients with impaired exchange in the assessment of patients with
ventricular function. $B r$ Heart $J 1985 ; 54: 321-8$.

14 Quyyumi AA, Mockus L, Wright C, Fox KM. Morphology of ambulatory ST segment changes in patients with varying severity of coronary artery disease: investigation of the frequency of nocturnal ischaemia and coronary spasm. Br Heart J 1985;53:186-93.

15 Mulcahy D, Keegan J, Lindsay D, Sparrow J, Park A, Wright C, Fox $\mathrm{K}$. Silent myocardial ischaemia in patients referred for coronary artery bypass surgery because of angina: a comparison with patients whose symptoms were well controlled on medical treatment. Br Heart J 1989; 61:496-501.

16 Mulcahy D, Keegan J, Sparrow J, Park A, Wright C, Fox KM. Ischemia in the ambulatory setting-the total ischemic burden: relation to exercise testing and investigative and therapeutic implications. J Am Coll Cardiol 1989;14:1166-72.

17 Aronow WS, Epstein S. Usefulness of silent myocardial ischemia detected by ambulatory electrocardiographic monitoring in predicting new coronary events in elderly monitoring in predicting new coronary

18 Tzivoni D, Gavish A, Zin D, et al. Prognostic significance of ischemic episodes in patients with previous myocardial ischemic episodes in patients with previo

19 Kennedy HL, Seiler SM, Sprague MK, et al. Relation of silent myocardial ischemia after coronary artery bypass grafting to angiographic completeness of revascularisation and long-term prognosis. Am J Cardiol 1990;65:14-22. 